\title{
Relationship between Hospital 30-Day Mortality Rates for Heart Failure and Patterns of Early Inpatient Comfort Care
}

\author{
Lena M. Chen, MD, MS 1,2,34*, Deborah A. Levine, MD1,2,4 Rodney Hayward, MD1,2,4 Margueritte Cox, MS, MGIST5, \\ Phillip J. Schulte, PhD ${ }^{6}$, Adam D. DeVore, MD, MHS ${ }^{5,7}$, Adrian Hernandez, MD, MHS ${ }^{5,7}$, Paul A. Heidenreich, MD, MS ${ }^{8}$, \\ Clyde Yancy, MD, MSc9, Gregg C. Fonarow, MD,10
}

\begin{abstract}
${ }^{1}$ Division of General Medicine, Department of Internal Medicine, University of Michigan, Ann Arbor, Michigan; ${ }^{2}$ VA Ann Arbor Healthcare System, Ann Arbor, Michigan; ${ }^{3}$ Center for Healthcare Outcomes \& Policy, University of Michigan, Ann Arbor, Michigan; ${ }^{4}$ Institute for Healthcare Policy \& Innovation, University of Michigan, Ann Arbor, Michigan; ${ }^{5}$ Duke Clinical Research Institute, Durham, North Carolina; ${ }^{6}$ Division of Biomedical Statistics and Informatics, Mayo Clinic, Rochester, Minnesota; ${ }^{7}$ Department of Medicine, School of Medicine, Duke University, Durham, North Carolina; ${ }^{8}$ VA Palo Alto Health Care System, Palo Alto, California; ${ }^{9}$ Feinberg School of Medicine, Northwestern University, Chicago, Illinois; ${ }^{10}$ Ahmanson-University of California at Los Angeles Cardiomyopathy Center, Geffen School of Medicine, University of California Los Angeles, Los Angeles, California.
\end{abstract}

BACKGROUND: The Centers for Medicare \& Medicaid Services rewards hospitals that have low 30-day riskstandardized mortality rates (RSMR) for heart failure (HF).

OBJECTIVE: To describe the use of early comfort care for patients with $\mathrm{HF}$, and whether hospitals that more commonly initiate comfort care have higher 30-day mortality rates.

DESIGN: A retrospective, observational study.

SETTING: Acute care hospitals in the United States.

PATIENTS: A total of 93,920 fee-for-service Medicare beneficiaries admitted with HF from January 2008 to December 2014 to 272 hospitals participating in the Get With The Guidelines-Heart Failure registry.

EXPOSURE: Early comfort care (defined as comfort care within 48 hours of hospitalization) rate.

MEASUREMENTS: A 30-day RSMR.
RESULTS: Hospitals' early comfort care rates were low for patients admitted for $\mathrm{HF}$, with no change over time ( $2.5 \%$ to $2.6 \%$, from 2008 to $2014, P=.56$ ). Rates varied widely ( $0 \%$ to $40 \%$ ), with $14.3 \%$ of hospitals not initiating comfort care for any patients during the first 2 days of hospitalization. Risk-standardized early comfort care rates were not correlated with RSMR (median RSMR $=10.9 \%$, 25 th to 75 th percentile $=10.1 \%$ to $12.0 \%$; Spearman's rank correlation $=0.13 ; P=.66$ ).

CONCLUSIONS: Hospital use of early comfort care for HF varies, has not increased over time, and on average, is not correlated with 30-day RSMR. This suggests that current efforts to lower mortality rates have not had unintended consequences for hospitals that institute early comfort care more commonly than their peers. Journal of Hospital Medicine 2018;13:170-176. () 2018 Society of Hospital Medicine $\mathrm{n}$ an effort to improve the quality of care delivered to heart failure (HF) patients, the Centers for Medicare \& Medicaid Services (CMS) publish hospitals' 30-day risk-standardized mortality rates (RSMRs) for HF. ${ }^{1}$ These mortality rates are also used by CMS to determine the financial penalties and bonuses that hospitals receive as part of the national Hospital Value-based Purchasing program. ${ }^{2}$ Whether or not these efforts effectively direct patients towards high-quality providers or motivate hospitals to provide better care, few would disagree with the overarching goal of decreasing the number of patients who die from HF.

\footnotetext{
*Address for correspondence: Lena M. Chen, MD, MS, University of Michigan Division of General Medicine, North Campus Research Complex, 2800 Plymouth Road, Building 16, Rm 407E, Ann Arbor, MI 48109-2800; Telephone: 734-936-5216; Fax: 734-936-8944; E-mail: lenac@umich.edu

Additional Supporting Information may be found in the online version of this article.
}

Received: March 22, 2017; Revised: July 7, 2017; Accepted: July 19, 2017

2018 Society of Hospital Medicine DOI 10.12788/jhm.2862
However, for some patients with chronic disease at the end of life, goals of care may change. The quality of days lived may become more important than the quantity of days lived. As a consequence, high-quality care for some patients at the end of life is associated with withdrawing life-sustaining or life-extending therapies. Over time, this therapeutic perspective has become more common, with use of hospice care doubling from 23\% to 47\% between 2000 and 2012 among Medicare beneficiaries who died. ${ }^{3}$ For a national cohort of older patients admitted with HF-not just those patients who died in that same year-hospitals' rates of referral to hospice are considerably lower, averaging 2.9\% in 2010 in a national study. ${ }^{4} \mathrm{Nev}$ ertheless, it is possible that hospitals that more faithfully follow their dying patients' wishes and withdraw life-prolonging interventions and provide comfort-focused care at the end of life might be unfairly penalized if such efforts resulted in higher mortality rates than other hospitals.

Therefore, we used Medicare data linked to a national HF registry with information about end-of-life care, to address 3 questions: (1) How much do hospitals vary in their rates of early 
comfort care and how has this changed over time; (2) What hospital and patient factors are associated with higher early comfort care rates; and (3) Is there a correlation between 30day risk-adjusted mortality rates for $\mathrm{HF}$ with hospital rates of early comfort care?

\section{METHODS}

\section{Data Sources}

We used data from the American Heart Association's Get With The Guidelines-Heart Failure (GWTG-HF) registry. GWTG-HF is a voluntary, inpatient, quality improvement registry ${ }^{5-7}$ that uses web-based tools and standard questionnaires to collect data on patients with $\mathrm{HF}$ admitted to participating hospitals nationwide. The data include information from admission (eg, sociodemographic characteristics, symptoms, medical history, and initial laboratory and test results), the inpatient stay (eg, therapies), and discharge (eg, discharge destination, whether and when comfort care was initiated). We linked the GWTG-HF registry data to Medicare claims data in order to obtain information about Medicare eligibility and patient comorbidities. Additionally, we used data from the American Hospital Association (2008) for hospital characteristics. Quintiles Real-World \& Late Phase Research (Cambridge, MA) serves as the data coordinating center for GWTG-HF and the Duke Clinical Research Institute (Durham, NC) serves as the statistical analytic center. GWTG-HF participating sites have a waiver of informed consent because the data are de-identified and primarily used for quality improvement. All analyses performed on this data have been approved by the Duke Medical Center Institutional Review Board.

\section{Study Population}

We identified 107,263 CMS-linked patients who were 65 years of age or older and hospitalized with HF at 348 fully participating GWTG-HF sites from February 17, 2008, to December 1, 2014. We excluded an additional 12,576 patients who were not enrolled in fee-for-service Medicare at admission, were transferred into the hospital, or had missing comfort measures only (CMO) timing information. We also excluded 767 patients at 68 sites with fewer than 30 patients. These exclusions left us with $93,920 \mathrm{HF}$ patients cared for at 272 hospitals for our final study cohort (Supporting Figure 1).

\section{Study Outcomes}

Our outcome of interest was the correlation between a hospital's rate of initiating early CMO for admitted HF patients and a hospital's 30-day RSMR for HF. The GWTG-HF questionnaire ${ }^{8}$ asks "When is the earliest physician/advanced practice nurse/ physician assistant documentation of comfort measures only?" and permits 4 responses: day 0 or 1 , day 2 or after, timing unclear, or not documented/unable to determine. We defined early $\mathrm{CMO}$ as $\mathrm{CMO}$ on day 0 or 1 , and late/no $\mathrm{CMO}$ as any other response. We chose to examine early comfort care because many hospitalized patients transition to comfort care before they die if the death is in any way predictable. Thus, if comfort care is measured at any time during the hospitalization, hospitals that have high mortality rates are likely to have high

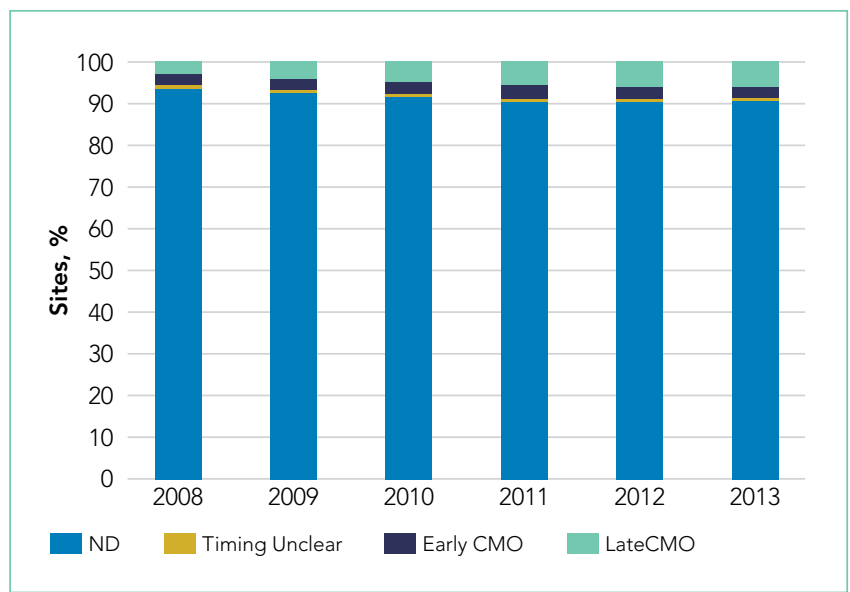

FIG 1. Trends in comfort care rates, stratified by timing of comfort care during a hospitalization, 2008-2013. ND indicates comfort measures not documented. $\mathrm{CMO}$ is comfort measures only, with early $\mathrm{CMO}$ defined as Day 0 or 1 in response to the question: "When is the earliest physician/APN/PA documentation of comfort measures only?" Late $\mathrm{CMO}$ is defined as CMO on Day 2 or later. For not documented, timing unclear, early $\mathrm{CMO}$, and late $\mathrm{CMO}, \mathrm{P}$ values are $<.001, .02, .56$, and $<.001$, respectively.

NOTE: Abbreviations: APN, advanced practice nurse; $\mathrm{CMO}$, comfort measures only; PA, physician assistant

comfort care rates. Therefore, we chose to use the more precise measure of early comfort care. We created hospital-level, risk-standardized early comfort care rates using the same risk-adjustment model used for RSMRs but with the outcome of early comfort care instead of mortality. 9,10

RSMRs were calculated using a validated GWTG-HF 30-day risk-standardized mortality model ${ }^{9}$ with additional variables identified from other GWTG-HF analyses. ${ }^{10}$ The 30 days are measured as the 30 days after the index admission date.

\section{Statistical Analyses}

We described trends in early comfort care rates over time, from February 17, 2008, to February 17, 2014, using the Cochran-Armitage test for trend. We then grouped hospitals into quintiles based on their unadjusted early comfort care rates. We described patient and hospital characteristics for each quintile, using $\chi^{2}$ tests to test for differences across quintiles for categorical variables and Wilcoxon rank sum tests to assess for differences across quintiles for continuous variables. We then examined the Spearman's rank correlation between hospitals' RSMR and risk-adjusted comfort care rates. Finally, we compared hospital-level RSMRs before and after adjusting for early comfort care.

We performed risk-adjustment for these last 2 analyses as follows. For each patient, covariates were obtained from the GWTG-HF registry. Clinical data captured for the index admission were utilized in the risk-adjustment model (for both RSMRs and risk-adjusted comfort care rates). Included covariates were as follows: age (per 10 years); race (black vs non-black); systolic blood pressure at admission $\leq 170$ (per $10 \mathrm{~mm} \mathrm{Hg}$ ); respiratory rate (per 5 respirations $/ \mathrm{min}$ ); heart rate $\leq 105$ (per 10 beats/ min); weight $\leq 100$ (per $5 \mathrm{~kg}$ ); weight $>100$ (per $5 \mathrm{~kg}$ ); blood urea nitrogen (per $10 \mathrm{mg} / \mathrm{dl}$ ); brain natriuretic peptide $\leq 2000$ (per $500 \mathrm{pg} / \mathrm{ml}$ ); hemoglobin 10-14 (per $1 \mathrm{~g} / \mathrm{dl}$ ); troponin abnormal 


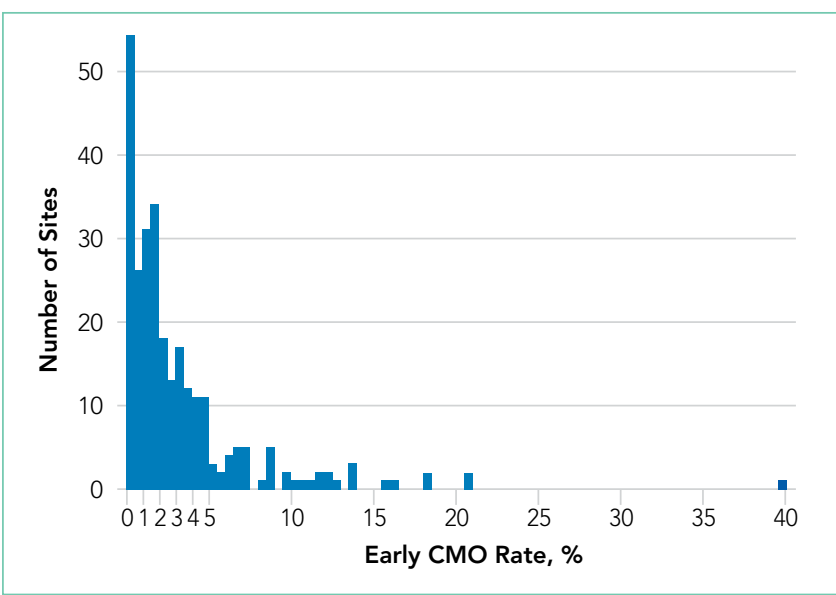

FIG 2. Hospital-level variation in comfort care rates. $\mathrm{CMO}$ is comfort measures only, with early CMO defined as Day 0 or 1 in response to the question: "When is the earliest physician/APN/PA documentation of comfort measures only?"

NOTE: Abbreviations: APN, advanced practice nurse; $\mathrm{CMO}$, comfort measures only; PA, physician assistant.

(vs normal); creatinine $\leq 1$ (per $1 \mathrm{mg} / \mathrm{dl}$ ); sodium 130-140 (per 5 $\mathrm{mEq} / \mathrm{l})$; and chronic obstructive pulmonary disease or asthma.

Hierarchical logistic regression modeling was used to calculate the hospital-specific RSMR. A predicted/expected ratio similar to an observed/expected (O/E) ratio was calculated using the following modifications: (1) instead of the observed (crude) number of deaths, the numerator is the number of deaths predicted by the hierarchical model among a hospital's patients given the patients' risk factors and the hospital-specific effect; (2) the denominator is the expected number of deaths among the hospital's patients given the patients' risk factors and the average of all hospital-specific effects overall; and (3) the ratio of the numerator and denominator are then multiplied by the observed overall mortality rate (same as $\mathrm{O} / \mathrm{E}$ ). This calculation is the method used by CMS to derive RSMRs. ${ }^{11}$ Multiple imputation was used to handle missing data in the models; 25 imputed datasets using the fully conditional specification method were created. Patients with missing prior comorbidities were assumed to not have those conditions. Hospital characteristics were not imputed; therefore, for analyses that required construction of risk-adjusted comfort care rates or RSMRs, we excluded 18,867 patients cared for at 82 hospitals missing hospital characteristics. We ran 2 sets of models for risk-adjusted comfort care rates and RSMRs: the first adjusted only for patient characteristics, and the second adjusted for both patient and hospital characteristics. Results from the 2 models were similar, so we present only results from the latter. Variance inflation factors were all $<2$, indicating the collinearity between covariates was not an issue.

All statistical analyses were performed by using SAS version 9.4 (SAS Institute, Cary, NC). We tested for statistical significance by using 2-tailed tests and considered $P$ values $<.05$ to be statistically significant.

\section{RESULTS}

Of the 272 hospitals included in our final study cohort, the observed median overall rate of early comfort care in this study

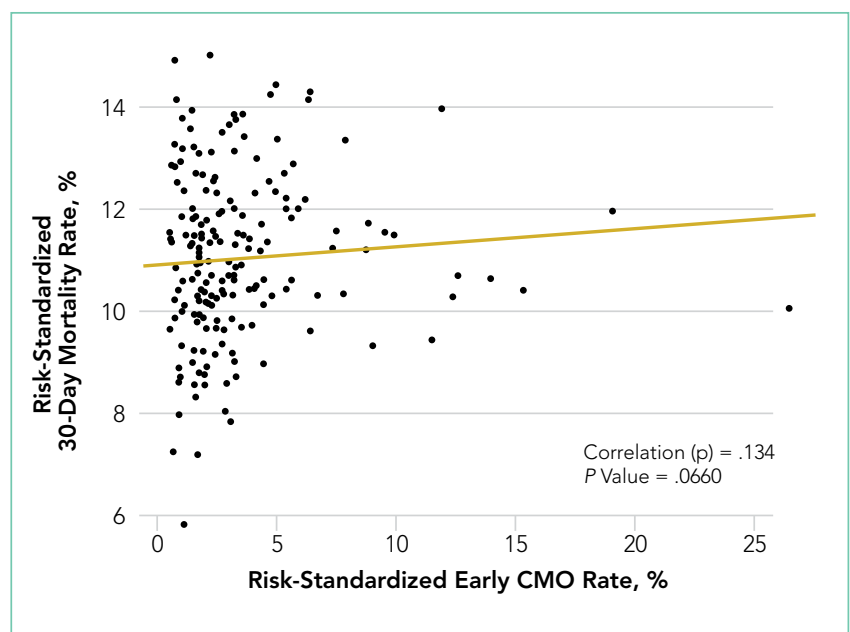

FIG 3. Correlation between hospitals' risk-standardized 30-day mortality rates and risk-adjusted comfort care rates. Each dot represents a single hospital. $\mathrm{CMO}$ is comfort measures only, with early $\mathrm{CMO}$ defined as Day 0 or 1 in response to the question: "When is the earliest physician/APN/PA documentation of comfort measures only?"

NOTE: Abbreviations: APN, advanced practice nurse; $\mathrm{CMO}$, comfort measures only; PA, physician assistant.

was $1.9 \%$ (25th to 75 th percentile: $0.9 \%$ to $4.0 \%$ ); hospitals varied widely in unadjusted early comfort care rates $(0.00 \%$ to $0.46 \%$ in the lowest quintile, and $4.60 \%$ to $39.91 \%$ in the highest quintile; Table 1).

The sociodemographic characteristics of the 93,920 patients included in our study cohort differed across hospital comfort care quintiles. Compared with patients cared for by hospitals in the lowest comfort care quintile, patients cared for by hospitals in the highest comfort care quintile were less likely to be male (44.6\% vs $46.7 \%, P=.0003)$, and less likely to be black ( $8.1 \%$ vs $14.0 \%)$, Asian (0.9\% vs $1.2 \%)$, or Hispanic (6.2\% vs $11.6 \%$; $P<$ .0001). Patients cared for at hospitals in the highest versus the lowest comfort care quintiles had slightly higher rates of prior stroke or transient ischemic attack (17.9\% vs $13.5 \%$; $P<.0001)$, chronic dialysis (4.7\% vs $2.9 \% ; P=.002)$, and depression $(12.8 \%$ vs $9.3 \%, P<.0001)$.

Compared to hospitals in the lowest comfort care quintile, hospitals in the highest comfort care quintile were as likely to be academic teaching hospitals (38.9\% vs $47.2 \%$; $P=.14$; Table 2). Hospitals in the highest comfort care quintiles were less likely to have the ability to perform surgical interventions, such as cardiac surgery $(52.6 \%$ vs $66.7 \%, P=.04)$ or heart transplants (2.5\% vs $12.1 \% ; P=.04)$.

Early comfort care rates showed minimal change from $2.60 \%$ in 2008 to $2.49 \%$ in 2013 ( $P=0.56$; Figure 1$)$. For this entire time period, there were a few hospitals that had very high early comfort care rates, but $90 \%$ of hospitals had comfort care rates that were $7.2 \%$ or lower. About $19.9 \%$ of hospitals (54 hospitals) initiated early comfort care on $0.5 \%$ or less of their patients admitted with HF; about half of hospitals initiated comfort care for $1.9 \%$ or fewer of their patients (Figure 2). There was a more even distribution of late $\mathrm{CMO}$ rate across hospitals (Supporting Figure 2). 


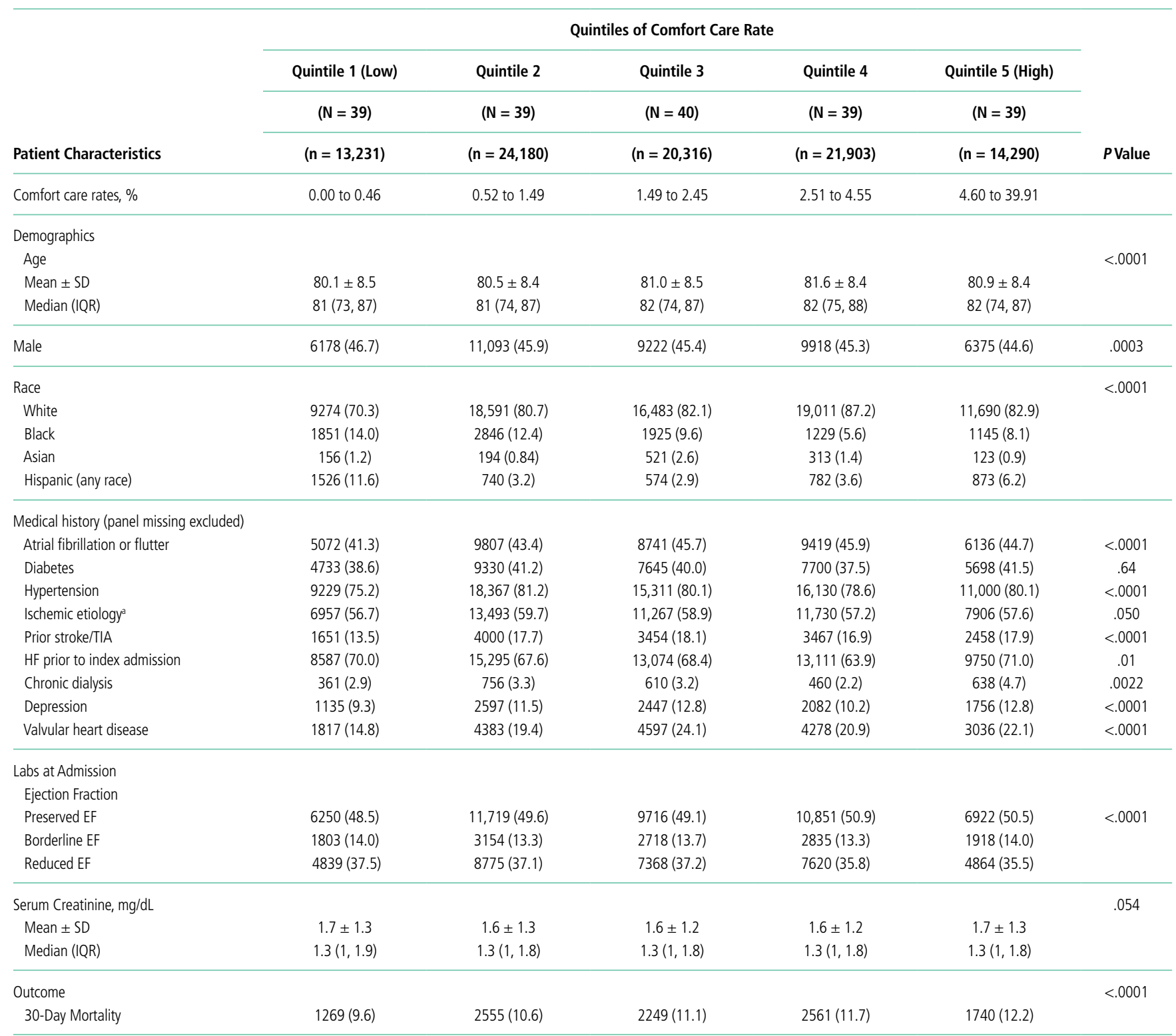

${ }^{a}$ Medical history of coronary artery disease, myocardial infarction, prior percutaneous coronary intervention, prior coronary artery bypass graft, or prior percutaneous coronary intervention and coronary artery bypass graft.

NOTE: $\mathrm{N}$ refers to number of hospitals; $\mathrm{n}$ refers to number of patients. Abbreviations: $\mathrm{EF}$, ejection fraction; HF, heart failure; IQR is interquartile range; SD, standard deviation; TIA, transient ischemic attack.

Hospitals' 30-day RSMR and risk-adjusted comfort care rates showed a very weak, but statistically insignificant positive correlation (Spearman's rank correlation $\rho=0.13, P=.0660$; Figure 3). Hospitals' 30-day RSMR before versus after adjusting for comfort care were largely similar (Supporting Figure 3). The median hospital-level RSMR was 10.9\%, 25th to 75th percentile, $10.1 \%$ to $12.0 \%$ (data not displayed). The mean difference between RSMR after comfort care adjustment, compared to before adjustment, was $0.001 \%$ (95\% confidence interval [Cl], $-0.014 \%$ to $0.017 \%$ ). However, for the 90 hospitals with comfort care rates of $1.9 \%$ (ie, the median) or above, mortality rates decreased slightly after comfort care adjustment (mean change of $-0.07 \% ; 95 \% \mathrm{Cl},-0.06$ to $-0.08 ; P<.0001)$. Patient-level RSMR decreased after excluding early comfort care patients, although the shape of the distribution remained the same (Supporting Figure 4).

\section{DISCUSSION}

Among a national sample of US hospitals, we found wide variation in how frequently health care providers deliver comfort care within the first 2 days of admission for HF. A minority of hospitals reported no early comfort care on any patients throughout the 6-year study period, but hospitals in the highest quintile initiated early comfort care rates for at least 1 in 
TABLE 2. Hospital Characteristics by Comfort Care Quintiles (at the Hospital Level)

\begin{tabular}{|c|c|c|c|c|c|c|}
\hline \multirow[b]{2}{*}{ Hospital Characteristics } & \multicolumn{5}{|c|}{ Quintiles of Comfort Care Rate } & \multirow[b]{2}{*}{$P$ Value } \\
\hline & $(n=5650)$ & $(n=13,420)$ & $(n=12,365)$ & $(n=10,885)$ & $(n=9300)$ & \\
\hline Comfort care rates, $\%$ & 0.00 to 0.46 & 0.52 to 1.49 & 1.49 to 2.45 & 2.51 to 4.55 & 4.60 to 39.91 & \\
\hline Mean \pm SD & $0.1 \pm 0.2$ & $1.0 \pm 0.2$ & $1.9 \pm 0.3$ & $3.5 \pm 0.6$ & $9.6 \pm 6.0$ & \\
\hline Median (IQR) & $0(0.0,0.2)$ & $1.0(0.82,1.2)$ & $1.9(1.7,2.2)$ & $3.4(3.0,4.0)$ & $7.3(5.9,11.8)$ & \\
\hline Academic/teaching hospital & $25(47.2)$ & $32(60.4)$ & $30(55.6)$ & $25(45.5)$ & $21(38.9)$ & .14 \\
\hline Number of beds & & & & & & .14 \\
\hline Mean \pm SD & $316.8 \pm 218.2$ & $403.8 \pm 248.8$ & $335.6 \pm 199.7$ & $305.4 \pm 194.4$ & $279 \pm 155.2$ & \\
\hline Median (IQR) & $279(150,410)$ & $355(227,550)$ & $312(177,440)$ & $270(165,405)$ & $253(161,368)$ & \\
\hline Heart transplants performed at site & $4(12.1)$ & $5(12.5)$ & $4(11.1)$ & $2(4.4)$ & $1(2.5)$ & .04 \\
\hline Rural Location & $5(9.3)$ & $5(9.1)$ & $5(9.3)$ & $4(7.3)$ & $8(15.4)$ & .44 \\
\hline Region & & & & & & .15 \\
\hline West & $6(11.1)$ & $4(7.3)$ & $7(13.0)$ & $10(18.2)$ & $9(16.7)$ & \\
\hline South & $26(48.2)$ & $22(40.0)$ & $13(24.1)$ & $16(29.1)$ & $18(33.3)$ & \\
\hline Midwest & $9(16.7)$ & $11(20.0)$ & $12(22.2)$ & $12(21.8)$ & $13(24.1)$ & \\
\hline Northeast & $13(24.1)$ & $18(32.7)$ & $22(40.7)$ & $17(30.9)$ & $14(25.9)$ & \\
\hline Length of stay & & & & & & $<.0001$ \\
\hline Mean \pm SD & $5.7 \pm 6.5$ & $5.4 \pm 5.3$ & $5.4 \pm 4.7$ & $5.1 \pm 4.8$ & $5.0 \pm 5.6$ & \\
\hline
\end{tabular}

NOTE: N refers to number of hospitals; $n$ refers to number of patients. Abbreviations: AMI, acute myocardial infarction; HF, heart failure; IQR is interquartile range; PTCA, percutaneous transluminal coronary angioplasty; SD, standard deviation; TIA, transient ischemic attack.

$20 \mathrm{HF}$ patients. Hospitals that were more likely to initiate early comfort care had a higher proportion of female and white patients and were less likely to have the capacity to deliver aggressive surgical interventions such as heart transplants. Hospital-level 30-day RSMRs were not correlated with rates of early comfort care.

While the appropriate rate of early comfort care for patients hospitalized with HF is unknown, given that the average hospital RSMR is approximately $12 \%$ for fee-for-service Medicare patients hospitalized with $\mathrm{HF}_{1}^{12}$ it is surprising that some hospitals initiated early comfort care on none or very few of their HF patients. It is quite possible that many of these hospitals initiated comfort care for some of their patients after 48 hours of hospitalization. We were unable to estimate the average period of time patients received comfort care prior to dying, the degree to which this varies across hospitals or why it might vary, and whether the length of time between comfort care initiation and death is related to satisfaction with end-of-life care. Future research on these topics would help inform providers seeking to deliver better end-of-life care. In this study, we also were unable to estimate how often early comfort care was not initiated because patients had a good prognosis. However, prior studies have suggested low rates of comfort care or hospice referral even among patients at very high estimated mortality risk. ${ }^{4}$ It is also possible that providers and families had concerns about the ability to accurately prognosticate, although several models have been shown to perform acceptably for patients hospitalized with $\mathrm{HF}^{13}$

We found that comfort care rates did not increase over time, even though use of hospice care doubled among Medicare beneficiaries between 2000 and 2012. By way of context, cancer-the second leading cause of death in the US-was responsible for $38 \%$ of hospice admissions in 2013, whereas heart disease (including but not limited to HF) — the leading cause of death- was responsible for $13 \%$ of hospice admissions. ${ }^{14}$ The 2013 American College of Cardiology Foundation and the American Heart Association guidelines for HF recommend consideration of hospice or palliative care for inpatient and transitional care. ${ }^{15}$ In future work, it would be important to better understand the drivers behind decisions around comfort care for patients hospitalized with HF.

With regards to the policy implications of our study, we found that on average, adjusting 30-day mortality rates for early comfort care was not associated with a change in hospital mortality rankings. For those hospitals with high comfort care rates, adjusting for comfort care did lower mortality rates, but 
the change was so small as to be clinically insignificant. CMS RSMR for HF excludes patients enrolled in hospice during the 12 months prior to index admission, including the first day of the index admission, acknowledging that death may not be an untoward outcome for such patients. ${ }^{16}$ Fee-for-service Medicare beneficiaries excluded for hospice enrollment comprised $1.29 \%$ of HF admissions from July 2012 to June $2015^{16}$ and are likely a subset of early comfort care patients in our sample, both because of the inclusiveness of chart review (vs claimsbased identification) and because we defined early comfort care as comfort care initiated on day 0 or 1 of hospitalization. Nevertheless, with our data we cannot assess to what degree our findings were due solely to hospice patients excluded from $\mathrm{CMS}^{\prime}$ current estimates.

Prior research has described the underuse of palliative care among patients with $\mathrm{HF}^{17}$ and the association of palliative care with better patient and family experiences at the end of life. ${ }^{18-}$ ${ }^{20}$ We add to this literature by describing the epidemiologyprevalence, changes over time, and associated factors-of early comfort care for $\mathrm{HF}$ in a national sample of hospitals. This serves as a baseline for future work on end-of-life care among patients hospitalized for HF. Our findings also contribute to ongoing discussion about how best to risk-adjust mortality metrics used to assess hospital quality in pay-for-performance programs. Recent research on stroke and pneumonia based on California data suggests that not accounting for do-not-resuscitate (DNR) status biases hospital mortality rates. ${ }^{21,22}$ Earlier research examined the impact of adjusting hospital mortality rates for DNR for a broader range of conditions. ${ }^{23,24}$ We expand this line of inquiry by examining the hospital-level association of early comfort care with mortality rates for $\mathrm{HF}$, utilizing a national, contemporary cohort of inpatient stays. In addition, while studies have found that DNR rates within the first 24 hours of admission are relatively high (median $15.8 \%$ for pneumonia; $13.3 \%$ for stroke), ${ }^{21,22}$ comfort care is distinct from DNR.

Our findings should be interpreted in the context of several potential limitations. First, we did not have any information about patient or family wishes regarding end-of-life care, or the exact timing of early comfort care (eg, day 0 or day 1 ). The initiation of comfort care usually follows conversations about end-of-life care involving a patient, his or her family, and the medical team. Thus, we do not know if low early comfort care rates represent the lack of such a conversation (and thus poor-quality care) or the desire by most patients not to initiate early comfort care (and thus high-quality care). This would be an important area for future research. Second, we included only patients admitted to hospitals that participate in GWTG$H F$, a voluntary quality improvement initiative. This may limit the generalizability of our findings, but it is unclear how our sample might bias our findings. Hospitals engaged in quality improvement may be more likely to initiate early comfort care aligned with patients' wishes; on the other hand, hospitals with advanced surgical capabilities are over-represented in our sample and these hospitals are less likely to initiate early comfort care. Third, we examined associations and cannot make conclusions about causality. Residual measured and unmeasured confounding may influence these findings.

In summary, we found that early comfort care rates for fee-for-service Medicare beneficiaries admitted for HF varies widely among hospitals, but median rates of early comfort care have not changed over time. On average, there was no correlation between hospital-level, 30-day, RSMRs and rates of early comfort care. This suggests that current efforts to lower mortality rates have not had unintended consequences for hospitals that institute early comfort care more commonly than their peers.

\section{Acknowledgments}

Dr. Chen and Ms. Cox take responsibility for the integrity of the data and the accuracy of the data analysis. Drs. Chen, Levine, and Hayward are responsible for the study concept and design. Drs. Chen and Fonarow acquired the data. Dr. Chen drafted the manuscript. Drs. Chen, Levin, Hayward, Cox, Fonarow, DeVore, Hernandez, Heidenreich, and Yancy revised the manuscript for important intellectual content. Drs. Chen, Hayward, Cox, and Schulte performed the statistical analysis. Drs. Chen and Fonarow obtained funding for the study. Drs. Hayward and Fonarow supervised the study. The authors thank Bailey Green, MPH, for the research assistance she provided. She was compensated for her work.

Disclosure: Dr. Fonarow reports research support from the National Institutes of Health, and consulting for Amgen, Janssen, Novartis, Medtronic, and St Jude Medical. Dr. DeVore reports research support from the American Heart Association, Amgen, and Novartis, and consulting for Amgen. The other authors have no relevant conflicts of interest. Dr. Chen was supported by a Career Development Grant Award (K08HS020671) from the Agency for Healthcare Research and Quality when the manuscript was being prepared. She currently receives support from the Department of Health and Human Services Office of the Assistant Secretary for Planning and Evaluation for her work there. She also receives support from the Blue Cross Blue Shield of Michigan Foundation's Investigator Initiated Research Program, the Agency for Healthcare Research and Quality (R01 HS024698), and the National Institute on Aging (P01 AG019783). These funding sources had no role in the preparation, review, or approval of the manuscript. The GWTG-HF program is provided by the American Heart Association. GWTG-HF has been funded in the past through support from Amgen, Medtronic, GlaxoSmithKline, Ortho-McNeil, and the American Heart Association Pharmaceutical Roundtable. These sponsors had no role in the study design, data analysis or manuscript preparation and revision.

\section{References}

1. Centers for Medicare \& Medicaid Services. Hospital Compare. https://www. medicare.gov/hospitalcompare/. Accessed on November 27, 2016

2. Centers for Medicare \& Medicaid Services. Hospital Value-based Purchasing. https://www.medicare.gov/hospitalcompare/data/hospital-vbp.html. Accessed August 30, 2017

3. Medicare Payment Advisory Comission. Report to the Congress: Medicare payment policy. 2014. http://www.medpac.gov/docs/default-source/reports/ mar14_entirereport.pdf. Accessed August 31, 2017.

4. Whellan DJ, Cox M, Hernandez AF, et al. Utilization of hospice and predicted mortality risk among older patients hospitalized with heart failure: findings from GWTG-HF. J Card Fail. 2012;18(6):471-477.

5. Hong Y, LaBresh KA. Overview of the American Heart Association "Get with the Guidelines" programs: coronary heart disease, stroke, and heart failure. Crit Pathw Cardiol. 2006;5(4):179-186.

6. LaBresh KA, Gliklich R, Liljestrand J, Peto R, Ellrodt AG. Using "get with the guidelines" to improve cardiovascular secondary prevention. Jt Comm J Qual Saf. 2003;29(10):539-550.

7. Hernandez AF, Fonarow GC, Liang L, et al. Sex and racial differences in the use of implantable cardioverter-defibrillators among patients hospitalized with heart failure. JAMA. 2007;298(13):1525-1532

8. Get With The Guidelines-Heart Failure. HF Patient Management Tool, October 2016. 
9. Eapen ZJ, Liang L, Fonarow GC, et al. Validated, electronic health record deployable prediction models for assessing patient risk of 30-day rehospitalization and mortality in older heart failure patients. JACC Heart Fail. 2013;1(3):245-251.

10. Peterson PN, Rumsfeld JS, Liang $L$, et al. A validated risk score for in-hospital mortality in patients with heart failure from the American Heart Association get with the guidelines program. Circ Cardiovasc Qual Outcomes. 2010;3(1):25-32.

11. Frequently Asked Questions (FAQs): Implementation and Maintenance of CMS Mortality Measures for AMI \& HF. 2007. https://www.cms.gov/Medicare/Quality-Initiatives-Patient-Assessment-Instruments/HospitalQualitylnits/downloads/HospitalMortalityAboutAMI_HF.pdf. Accessed August 30, 2017.

12. Suter LG, Li SX, Grady JN, et al. National patterns of risk-standardized mortality and readmission after hospitalization for acute myocardial infarction, heart failure, and pneumonia: update on publicly reported outcomes measures based on the 2013 release. J Gen Intern Med. 2014;29(10):1333-1340.

13. Lagu T, Pekow PS, Shieh MS, et al. Validation and comparison of seven mortality prediction models for hospitalized patients with acute decompensated heart failure. Circ Heart Fail. Aug 2016;9(8):e002912.

14. National Hospice and Palliative Care Organization. NHPCO's facts and figures: hospice care in america. 2015. https://www.nhpco.org/sites/default/ files/public/Statistics_Research/2015_Facts_Figures.pdf. Accessed August 30, 2017.

15. Yancy CW, Jessup M, Bozkurt B, et al. 2013 ACCF/AHA guideline for the management of heart failure: executive summary: a report of the American College of Cardiology Foundation/American Heart Association Task Force on practice guidelines. Circulation. 2013;128(16):1810-1852.
16. Centers for Medicare \& Medicaid Services. 2016 Condition-Specific Measures Updates and Specifications Report Hospital-Level 30-Day Risk-Standardized Mortality Measures. https://www.qualitynet.org/dcs/ ContentServer?c=Page\&pagename $=$ QnetPublic\%2FPage\%2FOnetTier3\&cid=1228774398696. Accessed August 30, 2017.

17. Bakitas M, Macmartin M, Trzepkowski K, et al. Palliative care consultations for heart failure patients: how many, when, and why? J Card Fail. 2013;19(3): 193-201.

18. Wachterman MW, Pilver C, Smith D, Ersek M, Lipsitz SR, Keating NL. Quality of End-of-Life Care Provided to Patients With Different Serious IIlnesses. JAMA Intern Med. 2016;176(8):1095-1102.

19. Wright AA, Zhang $B$, Ray $A$, et al. Associations between end-of-life discussions, patient mental health, medical care near death, and caregiver bereavement adjustment. JAMA. 2008;300(14):1665-1673.

20. Rogers JG, Patel CB, Mentz RJ, et al. Palliative care in heart failure: results of a randomized, controlled clinical trial. J Card Fail. 2016;22(11):940.

21. Kelly AG, Zahuranec DB, Holloway RG, Morgenstern LB, Burke JF. Variation in do-not-resuscitate orders for patients with ischemic stroke: implications for national hospital comparisons. Stroke. 2014;45(3):822-827.

22. Walkey AJ, Weinberg J, Wiener RS, Cooke CR, Lindenauer PK. Association of Do-Not-Resuscitate Orders and Hospital Mortality Rate Among Patients With Pneumonia. JAMA Intern Med. 2016;176(1):97-104.

23. Bardach N, Zhao S, Pantilat S, Johnston SC. Adjustment for do-not-resuscitate orders reverses the apparent in-hospital mortality advantage for minorities. Am J Med. 2005;118(4):400-408.

24. Tabak YP, Johannes RS, Silber JH, Kurtz SG. Should Do-Not-Resuscitate status be included as a mortality risk adjustor? The impact of DNR variations on performance reporting. Med Care. 2005;43(7):658-666. 